\title{
A UNIQUENESS THEOREM FOR BELTRAMI EQUATIONS
}

\author{
L. AHLFORS AND G. WEILL ${ }^{1}$
}

1. In the theory of quasiconformal mappings the Beltrami equation

$$
f_{z}=\mu f_{z}
$$

with complex $\mu,|\mu| \leqq k<1$, plays an important role. In particular, one is led to consider the special case where $\mu$ is given in $|z|<1$ by an expression

$$
\mu(z)=\bar{q}(z)(1-z \bar{z})^{2},
$$

where $q$ is analytic and subject to the condition

$$
\left|q(1-z \bar{z})^{2}\right| \leqq k<1 .
$$

Elementary classical methods yield a particular solution of (1.1). It is the purpose of this note to show that this solution is univalent, and that $q$ is uniquely determined by the boundary values of $f$. Related questions occur in a paper by L. Bers [2] which was written independently of the present authors.

2. If we rewrite $(1.1)$ as

$$
\bar{f}_{z}=q(z)(1-z \bar{z})^{2} \bar{f}_{\bar{z}}
$$

it is classical that $\bar{f}$ may be determined from the general solution of the ordinary differential equation

$$
d \bar{z} / d z=-q(z)(1-z \bar{z})^{2}
$$

where $z$ and $\bar{z}$ are regarded as independent variables. By the general theory, if $v_{1}$ and $v_{2}$ are linearly independent solutions of the associated linear equation

$$
v^{\prime \prime}=q v
$$

then (2.1) admits the solution

$$
\bar{f}=\frac{\bar{z} v_{2}+(1-z \bar{z}) v_{2}^{\prime}}{\bar{z} v_{1}+(1-z \bar{z}) v_{1}^{\prime}} .
$$

We shall show that $f$, as determined by (2.4), is univalent in $|z|<1$, from which it follows that all solutions of (1.1) can be expressed as analytic functions of $f$.

Received by the editors November 28, 1961.

${ }^{1}$ Work supported by the Air Force Office of Scientific Research, under Contract AF 49(638)-574. 
3. The expression (2.4) leads us to surmise that $\bar{f}$ has the boundary values $v_{2} / v_{1}$. Under the condition $\left|q(1-z \bar{z})^{2}\right|<1 \mathrm{Z}$. Nehari [3] has proved that $v_{2} / v_{1}$ is univalent in $|z|<1$. With the stronger assumption (1.3) we show that it remains continuous and univalent on the circumference $|z|=1$. The proof rests on the following lemma:

LEмMA. Let $v$ be the solution of (2.3) which is normalized by $v(0)=1$, $v^{\prime}(0)=0$. Under the condition (1.3) v satisfies

$$
\begin{gathered}
\operatorname{Re}\left(z v^{\prime} / v\right) \geqq-k z \bar{z}(1-z \bar{z})^{-1} \\
|v| \geqq(1-z \bar{z})^{k / 2} .
\end{gathered}
$$

Proof. It is sufficient to consider positive real values of $\boldsymbol{z}$, for $v_{\alpha}(z)=e^{-i \alpha} v\left(z e^{i \alpha}\right)$ and $q_{\alpha}(z)=e^{2 i \alpha} q\left(z e^{i \alpha}\right)$ satisfy the same conditions as $v$ and $q$. From (2.3) we obtain

$$
\int_{0}^{r} \bar{v} v^{\prime \prime} d x=\bar{v} v^{\prime}-\int_{0}^{r}\left|v^{\prime}\right|^{2} d x=\int_{0}^{r} q|v|^{2} d x
$$

and hence

$$
\operatorname{Re}\left(\bar{v} v^{\prime}\right) \geqq \int_{0}^{r}\left|v^{\prime}\right|^{2} d x-\int_{0}^{r}\left|q v^{2}\right| d x .
$$

If $u=\left(1-x^{2}\right)^{k / 2}$ one verifies that

$$
u^{\prime \prime} / u \leqq-k\left(1-x^{2}\right)^{-2} \leqq-|q|
$$

Therefore,

$$
\begin{aligned}
& -\int_{0}^{r}\left|q v^{2}\right| d x \geqq \int_{0}^{r}\left(u^{\prime \prime} / u\right)|v|^{2} d x \\
& =\left(u^{\prime} / u\right)|v|^{2}+\int_{0}^{r}\left(u^{\prime} / u\right)^{2}|v|^{2} d x-2 \int_{0}^{r}\left(u^{\prime} / u\right) \operatorname{Re}\left(\bar{v} v^{\prime}\right) d x \\
& =\left(u^{\prime} / u\right)|v|^{2}+\int_{0}^{r}\left|\left(u^{\prime} / u\right) v-v^{\prime}\right|^{2} d x-\int_{0}^{r}\left|v^{\prime}\right|^{2} d x,
\end{aligned}
$$

and it follows from (3.4) and (3.6) that

$$
\operatorname{Re}\left(\bar{v} v^{\prime}\right) \geqq\left(u^{\prime} / u\right)|v|^{2} .
$$

But this is precisely (3.1), and (3.2) follows by integration from 0 to $r$.

4. In (2.4) we normalize $v_{1}$ by $v_{1}(0)=1, v_{1}^{\prime}(0)=0$, as in the lemma, and $v_{2}$ by $v_{2}(0)=0, v_{2}^{\prime}(0)=1$. Then $v_{2}^{\prime} v_{1}-v_{1}^{\prime} v_{2}=1$, and $g=v_{2} / v_{1}$ has the derivative $g^{\prime}=v_{1}^{-2}$. The lemma yields $\left|g^{\prime}\right| \leqq(1-z \bar{z})^{-k}$, and be- 
cause $k<1$ it is immediate that $g$ has a continuous extension to $|z|$ $=1$.

Observe that $f$ is infinite at $z=0$, but at no other point. To determine the boundary behavior we form

$$
\bar{f}(z)-g(z)=z v_{1}^{-2}(1-z \bar{z})\left(z \bar{z}+(1-z \bar{z}) z v_{1}^{\prime} / v_{1}\right)^{-1} .
$$

By use of the lemma,

$$
\left|z \bar{z}+(1-z \bar{z}) z v_{1}^{\prime} / v_{1}\right| \geqq z \bar{z}+(1-z \bar{z}) \operatorname{Re}\left(z v_{1}^{\prime} / v_{1}\right) \geqq(1-k) z \bar{z}
$$
and consequently

$$
|\bar{f}-g| \leqq|z|^{-1}(1-k)^{-1}(1-z \bar{z})^{1-k} .
$$

We conclude that $\bar{f}$ has indeed the same boundary values as $g=v_{2} / v_{1}$. Let us also note that

$$
f_{z}=\left(z \bar{v}_{1}+(1-z \bar{z}) \bar{v}_{1}^{\prime}\right)^{-2} .
$$

Application of (4.2) and (3.2) leads to the estimate

$$
\left|f_{z}\right| \leqq|z|^{-2}(1-k)^{-2}\left|g^{\prime}\right| \text {. }
$$

5. From these considerations it is a short step to the main theorem:

Theorem A. The functions

$$
f=\frac{z \bar{v}_{2}+(1-z \bar{z}) \bar{v}_{2}^{\prime}}{z \bar{v}_{1}+(1-z \bar{z}) \bar{v}_{1}^{\prime}} \quad \text { and } \quad \bar{g}=\bar{v}_{2} / \bar{v}_{1}
$$

are both continuous and univalent in $|z| \leqq 1$. They map the unit disk onto complementary regions of the extended plane whose common boundary is a Jordan curve with zero area.

Proof. Define $F$ by $F(z)=f(z)$ for $|z| \leqq 1, F(z)=\bar{g}(1 / \bar{z})$ for $|z|>1$. Then $F$ is a continuous mapping from the extended plane to the extended plane which satisfies the Beltrami equation

$$
F_{\bar{z}}=\mu F_{z}
$$

with $\mu=\bar{q}(1-z \bar{z})^{2}$ for $|z|<1, \mu=0$ for $|z|>1$. Furthermore, because $g$ is bounded and univalent (by Nehari's theorem) $g^{\prime}$ is square integrable, and the estimate (4.5) permits us to conclude that $F_{z}$ is locally square integrable (with the obvious modifications at 0 and $\infty$ ). By the generalized Riemann mapping theorem (see Ahlfors-Bers [1]) there exists a solution $w$ of the equation (5.2) which maps the sphere homeomorphically onto itself, and $F$ must be a rational function of $w$. Because $F$ is infinite at only one point it must be a linear function of 
$w$, and hence it is univalent. Together with the fact that null sets are mapped on null sets this proves the theorem.

According to familiar properties of quasiconformal mappings the proof yields the additional information that $f, g, f^{-1}$ and $g^{-1}$ satisfy Hölder conditions of order $1 / K=(1-k) /(1+k)$.

6. The uniqueness theorem is an almost trivial consequence:

TheOREM B. The function $q$ is uniquely determined by the boundary values of $f$.

Proof. Suppose that $f_{1}, f_{2}$ are determined by $q_{1}, q_{2}$, and that $f_{1}=f_{2}$ on $|z|=1$. The corresponding $g_{1}, g_{2}$ satisfy $g_{1}^{-1} g_{2}=z$ for $|z|=1$, and hence also for $|z|<1$. Thus $g_{1}$ and $g_{2}$ are identical, and the same must be true of their Schwarzian derivatives $\left\{g_{1}, z\right\}=-2 q_{1},\left\{g_{2}, z\right\}$ $=-2 q_{2}$.

\section{REFERENCES}

1. L. Ahlfors and L. Bers, Riemann's mapping theorem for variable metrics, Ann. of Math. 72 (1960), 385-404.

2. L. Bers, Correction to "Spaces of Riemann surfaces as bounded domains," Bull. Amer. Math. Soc. 67 (1961), 465-466.

3. Z. Nehari, The Schwarzian derivative and schlicht functions, Bull. Amer. Math. Soc. 55 (1949), 545-551.

Harvard UnIVersity 